\title{
Primary pulmonary hypertension and functional hyposplenism
}

\author{
Gerard Fahy, Killian Robinson, Binayak Deb and Ian Graham
}

\author{
Department of Cardiology, Meath and Adelaide Hospitals, Dublin, Ireland
}

\begin{abstract}
Summary: Primary pulmonary hypertension is a rare disease of unknown cause. Functional hyposplenism is characterized by the appearance of abnormal circulating erythrocytes despite the presence of a spleen and is associated with a variety of disease states. A case of primary pulmonary hypertension associated with functional hyposplenism is described in this report.
\end{abstract}

\section{Introduction}

Primary pulmonary hypertension is a rare disease of unknown aetiology usually affecting young adult females. It is characterized by progressive right heart failure typically causing death within 3 years of diagnosis. ${ }^{1}$ Pathologically it is recognized by changes in muscular pulmonary arteries consisting of medial hypertrophy intimal proliferation and plexiform lesions. ${ }^{2}$

Functional hyposplenism is an uncommon condition characterized by the appearance of abnormal erythrocytes (Howell-Jolly bodies, target cells, acanthocytes, siderocytes) and platelets in the blood despite the presence of a spleen. It is associated with sickle cell disease, coeliac disease, inflammatory bowel disease, amyloidosis and splenic artery thrombosis. ${ }^{3}$

We now report a patient with primary pulmonary hypertension and functional hyposplenism. Possible explanations for this previously undescribed association are discussed.

\section{Case report}

A 39 year old female presented with a 3 week history of progressive dyspnoea and chest pain. The history was otherwise unremarkable. Physical examination revealed features of right heart failure. The chest X-ray demonstrated an enlarged main pulmonary artery. Isotope lung scanning revealed multiple mismatches of ventilation and perfusion. The full blood count was normal apart from a mean corpuscular volume (MCV) of $101 \mathrm{fl}$. A blood film was not examined at this time. Doppler

Correspondence: K. Robinson, M.D., M.R.C.P., The Adelaide Hospital, Peter Street, Dublin 8, Ireland. Accepted: 14 October 1991 studies of the veins of the lower limbs were unremarkable. A diagnosis of right heart failure secondary to multiple pulmonary emboli of unknown origin was made and she was treated with anticoagulants and diuretics.

Two years later she presented with symptoms and signs of worsening right heart failure. Cardiac catheterization, after treatment of the heart failure, revealed the following resting pressures: right atrium (mean) $10 \mathrm{mmHg}$, right ventricle $38 / 11$ $\mathrm{mmHg}$, pulmonary artery $39 / 25$ (mean $31 \mathrm{mmHg}$ ), pulmonary artery wedge $7 \mathrm{mmHg}$. Cardiac output was $2.55 \mathrm{l} / \mathrm{min}$. Further isotope lung scanning revealed persistent mismatches of ventilation and perfusion. Bilateral lower limb venography was normal. Ultrasound examination of the abdomen revealed hepatomegaly with dilated hepatic veins consistent with congestive failure but the spleen was of normal size. Incidental findings included the presence of gallstones and a uterine fibroid. Blood film demonstrated Howell-Jolly bodies, anisocytes, spherocytes, macrocytes and macrothrombocytes. The haemoglobin measured $19.6 \mathrm{~g} / \mathrm{dl}$. The total red cell count was $5.91 \times 10^{12} / 1$ which was above the normal range of $3.8-5.8 \times 10^{12} / 1$. The haematocrit was $62.8 \%$ (normal range $37-47 \%$ ). Platelet count was normal as was the activated partial thromboplastin time (APTT). Antithrombin III levels were within the normal range. Protein $\mathrm{S}$ and $\mathrm{C}$ estimations were not performed.

Arterial blood gas analysis showed a $\mathrm{PO}_{2}$ of $9.57 \mathrm{kPa}$ and the $\mathrm{PCO}_{2}$ was $3.79 \mathrm{kPa}$. Serum erythropoietin was $50 \mathrm{mU} / \mathrm{ml}$ (normal $=5-30 \mathrm{mU} / \mathrm{l}$ ). MCV was $106 \mathrm{fl}$. Serum vitamin $B_{12}$, folic acid and thyroid function were all normal. Serum liver function tests were abnormal: total bilirubin elevated to $24 \mu \mathrm{mol} / 1$ (normal range $0-17 \mu \mathrm{mol}$ ), aspartate transaminase $42 \mathrm{IU} / 1$ (range 7-40 IU/1). These were compatible with liver congestion and 
fluctuated during the clinical course in relation to the severity of heart failure. Antinuclear antibody was positive at a titre of $1 / 160$ but tests for antibodies to double-stranded DNA, extractable nuclear antigen, smooth muscle, mitochondria and reticulin were negative. Rheumatoid factor was absent. Serum was not examined for the presence of antiphospholipid antibodies.

Over the next 4 years right heart failure gradually worsened. She was finally admitted suffering from atrial fibrillation and hypotension, developed pulmonary oedema and died.

At autopsy, the heart showed marked right ventricular hypertrophy (weight $120 \mathrm{~g}$ ). There was evidence of previous ischaemia (widespread foci of fibrosis) with recent infarction involving the anterior papillary muscle of the right ventricle. There was also extensive interstitial fibrosis of the left ventricle. The coronary arteries were normal.

Histological examination of the lungs revealed fibrous proliferation of the intima of small arteries and arterial dilation lesions including angiomatoid and plexiform lesions. There were also many organized and recanalized thrombi.

Grossly, the spleen was markedly atrophic weighing $4 \mathrm{~g}$. Histological examination showed sinusoidal atrophy with collapse of the capsule and trabeculae. Parenchymal vessels showed intimal proliferation. One of the smaller splenic arteries showed a recanalized thrombous. Gamna-Gandi bodies were also evident. These features suggested acquired splenic atrophy. The small intestinal mucosa was normal. The liver showed severe centrilobular venous congestion progressing to fibrosis.

\section{Discussion}

Primary pulmonary hypertension is a rare disease characterized by unexplained elevation of pulmonary blood pressure. It is typified by a relatively brief clinical course which usually terminates in right heart failure within a short time following diagnosis. Hyposplenism is an uncommon condition most often related to splenectomy. Occasionally hyposplenic changes are noted in the peripheral blood when the spleen is present; this is then termed functional hyposplenism.

This patient had pulmonary hypertension, the cause of which was uncertain during life. Although the presence of mismatched ventilation and perfusion suggested thromboembolic disease, postmortem examination revealed the diagnosis of primary pulmonary hypertension. ${ }^{4}$ In addition, thrombosis in situ was noted in the pulmonary blood vessels. This would have explained the abnormal ventilation perfusion scans ${ }^{5}$ seen during the earlier part of the clinical course.
Serial ultrasound examinations had consistently documented normal splenic size from the time of presentation until 8 months before death. By the time of autopsy, however, the spleen had shrunk to weigh only $4 \mathrm{~g}$. Despite the normal splenic size, function was impaired as reflected by the appearance of abnormal erythrocytes and platelets in the blood.

It is unclear in this case whether the development of primary pulmonary hypertension caused functional hyposplenism or if some other disease caused both pulmonary hypertension and functional hyposplenism. The first possibility could be explained by the complications of primary pulmonary hypertension which could be expected to cause splenic ischaemia, hypofunction and eventually atrophy: namely, reduced cardiac output, splenic venous hypertension, hypoxia and polycythaemia ${ }^{6}$ These factors would also predispose to splenic arterial thrombosis which would further compromise splenic perfusion and function. ${ }^{3}$ However, if these were the only causes of functional hyposplenism in this patient, one would expect previous reports of functional hyposplenism in patients with primary pulmonary hypertension and other causes of cor pulmonale.

An alternative is that another disease caused both pulmonary hypertension and functional hypo splenism. The only diseases known to cause both are systemic lupus erythematosus ${ }^{7,8}$ and rheumatoi arthritis. ${ }^{9,10}$ While this patient had a positive antinuclear antibody titre of $1 / 160$ this is not uncommon in primary pulmonary hypertension ${ }^{11}$ and there were no other immunological or clinical features of these diseases.

An underlying thrombotic diathesis is suggested by the presence of splenic arterial thrombosis, myocardial infarction with normal epicardial coronary arteries, and possibly by multiple pulmonary arterial thromboses. Antithrombin 3 levels were normal but protein $S$ and $C$ estimations were not performed. Antiphospholipid antibodies have been associated with pulmonary hypertension ${ }^{12}$ and splenic infarction ${ }^{13}$ but not functional hyposplenism. Unfortunately serum was not tested for the presence of antiphospholipid antibodies in this patient. This patient, however, had no history of recurrent spontaneous abortions and had a consistently normal APTT and platelet count making the diagnosis of the primary antiphospholipid syndrome unlikely.

\section{Acknowledgement}

We are grateful to Mrs Betty Turner who typed the manuscript and to the Department of Pathology, St James' Hospital, Dublin 8, for their help with the interpretation of the pathology findings. 


\section{References}

1. Wallcott, G., Burchell, H.B. \& Brown, A.L. Jr. Primary pulmonary hypertension. Am J Med 1970, 49: 70-79.

2. Edwards, W.D. \& Edwards, J.E. Clinical primary pulmonary hypertension. Circulation 1977, 5: 884-888.

3. Spencer, R.P., Dhawan, V., Suresh, K. et al. Causes and temporal sequence of onset of functional asplenia in adults. Clin Nucl Med 1978, 3: 17-19.

4. Hatano, S. \& Strasser, T. (eds). Primary pulmonary hypertension. Report on a WHO meeting. World Health Organisation, Geneva, 1975.

5. Wilson, A.G., Harris, C.N., Lavender, J.P. \& Oakley, C.M. Perfusion lung scanning in obliterative pulmonary hypertension. Br Heart J 1973, 35: 917-930.

6. Pearson, H.A., Schiebler, G.L. \& Spencer, R.P. Functional hyposplenia in cyanotic congenital heart disease. Pediatrics 1971, 48: 277-280.

7. Nair, S.S., Askari, A.D., Popelka, C.G. \& Kleinerman, J.F. Pulmonary hypertension and systemic lupus erythematosus. Arch Int Med 1980, 140: 109-111.
8. Dillon, A.M., Stein, H.B. \& English, R.A. Splenic atrophy in systemic lupus erythematosus. Ann Int Med 1982, 96: 40-43.

9. Onodera, S. \& Hill, J.R. Pulmonary hypertension: Report of a case in association with rheumatoid arthritis. Ohio Med $J$ 1965, 61: 141-144.

10. Williams, B.D., Pussell, B.A., Lockwood, C.M. \& Cotton, C. Defective reticuloendothelial system function in rheumatoid arthritis. Lancet 1979, 1: 1311-1314.

11. Hughes, J.D. \& Rubin, L.J. Primary pulmonary hypertension. An analysis of 28 cases and a review of the literature. Medicine Baltimore 1986, 65: 56-72.

12. Jeffrey, P.J., Asherson, R.A. \& Reef, P.J. Recurrent deep vein thrombosis, thromboembolic pulmonary hypertension and the 'primary antiphospholipid syndrome'. Clin Exp Rheumatol 1989, 7: 567-569.

13. Arnold, N.H. \& Schrieber, L. Splenic and renal infarction in systemic lupus erythematosus: association with anticardiolipin antibodies. Clin Rheumatol 1988, 7: 406-410. 